\title{
Numerical Simulation of the Carbon Fiber Composite Material Front Floor of Car by PAM-RTM
}

\author{
JI Junyang ${ }^{1}$, LIU Tingting ${ }^{1}$, FU Chao ${ }^{1}$, LI Zhong ${ }^{1}$, GAN Xuihui ${ }^{* 1}$ \\ ${ }^{1}$ College of Mechanical Engineering, Donghua University, Shanghai 201620; Engineering \\ Research Center of Textile Equipment, Ministry of Education, Shanghai 201620
}

Key words: Car floor; VARI; PAM-RTM; Runner; Filling

\begin{abstract}
By simulating the runner of the VARI molding process with PAM-RTM about the front floor of the car, examines the different injection methods, different influence of distance of hose guide for filling time, analyzing the resin flow patterns, the effects of the mold cavity pressure distribution and the overflow outlet location on the floor molding, and got the shortest time of program filling of the floor . From a test about VARI molding of the floor on the former basis, got the filling time 820s and it is consistent with simulation results 982s. The research results have important significance for research and application of carbon fiber composites.
\end{abstract}

\section{Introduction}

Vacuum assists resin injection (VARI) is a effective method of the application of large composite parts in National Defense ${ }^{[1-2]}$. The molding process relies on the technological conditions of permeability of fiber, injection pressure, resin viscosity, mold temperature, the shape and size of injection hole, the position of exhaust port and so forth in the process of resin filling ${ }^{[3]}$. These parameters that are required by the forming of VARI have coupling relationship and its influence is complicated. In the previous produce of composite materials, technique parameters setting mainly rely on "trial-and-error method", but it needs to consume the huge effort and resources which increase the cost of molding process. Otherwise, the injection holes of molds and the position of outlet have obvious influence on the shape of parts, therefore, the more reasonable holes of injection and the position of exhaust port can be found only by constantly experiments.

Up to now, the research on the numerical simulation of the carbon fiber with VARI process is rare. This paper uses finite-element method to simulate the VARI process of front floor of car, and by designing the positions of injection and the position of extraction ports of different resins we study how the flowing rules, filling time, the distribution of resin pressure in the mold influences the filling process, which thereby shortens the forming cycle and increases yield and reduces the cost of products.

\section{Mathematical model}

The VARI process of the forming of Composite material in the filling process is a complicated physical and chemical process. Exothermic phenomena occurs in the reaction, which causes the change of temperature in the mold. It will further affect the physical and chemical characteristics of rein, such as resin viscosity.

However, in consideration of the slow chemical reaction with short filling time for the forming process, this paper does not take the effect of chemical reaction on the forming process into account, while it is considered as a physical process.

Carbon fiber reinforcement is a kind of porous medium, and it is complex for resin to flow within it. The report that the simulation relating tho the real flow of resin int the reinforcement is rare at present ${ }^{[4]}$. In the simulation, the filling process of resin is considered as laminar flow of Newtonian liquid in the porous medium. Thus, we apply the Darcy's Law to simulate and analyze ${ }^{[5]}$. With the issues studied in this paper, carbon fiber reinforcement can be considered as it does not deform with the flow of resin, but it is regarded as rigid body. The filling pressure is small, so the 
resin can be regarded as incompressible Newtonian fluid; Compared to the driving force of the resin flow, the surface tension is very small and can be ignored, the resistance of cavity wall to resin is very small and can be ignored ${ }^{[6]}$. Through the computer numerical simulation of VARI molding process, we can get the resin flow pattern in the process of injection, the flow of the fluid pressure distribution as well as any time forward position, thus to predict the forming process of mold filling time, forming defects, etc.

The Darcy rules of fluid flow in porous media of anisotropy ${ }^{[7]}$ :

$$
\bar{V}=-\frac{1}{\mu} K \bullet \nabla p
$$

$\bar{V}$ is volume flow rate and $\mu$ is fluid viscosity.p is fluid pressure and k is medium permeability.

The permeability of preforming body is a key parameter of Darcy's law, which will directly affect the speed of resin flow, thus affecting the molding defects. As one of the important parameters of flow simulation, the permeability $\boldsymbol{K}$ plays a decisive role on the flow characteristics of resin ${ }^{[8]}$.Because the front floor thickness direction of the car in this article, compared with the length and width directions, is small, and is negligible. The resin flow is mainly in the $\mathrm{X}$ and $\mathrm{Y}$ directions, so it can be condidered as 2D plane structure. The 2D plane darcy's law is:

$$
\begin{gathered}
\left\{\begin{array}{l}
u \\
v
\end{array}\right\}=-\frac{1}{\mu}\left(\begin{array}{cc}
K x x & K x y \\
K y x & K y y
\end{array}\right)\left\{\begin{array}{l}
\frac{\partial P}{\partial x} \\
\frac{\partial P}{\partial y}
\end{array}\right\} \\
\frac{\partial u}{\partial x}+\frac{\partial v}{\partial y}=0
\end{gathered}
$$

Combine (3) and (2), in a control body, perform integral on (2) ${ }^{[9]}$; VARI molding process is described in the basic control equation:

$$
\iint \frac{1}{\mu}\left[\begin{array}{ll}
n_{x} & n_{y}
\end{array}\right]\left[\begin{array}{ll}
K x x & K x y \\
K y x & K y y
\end{array}\right]\left\{\begin{array}{l}
\frac{\partial p}{\partial x} \\
\frac{\partial p}{\partial y}
\end{array}\right\} d s=0
$$

$\boldsymbol{n}_{\boldsymbol{x}}, \boldsymbol{n}_{\boldsymbol{y}}$ are the direction vector components on the surface.

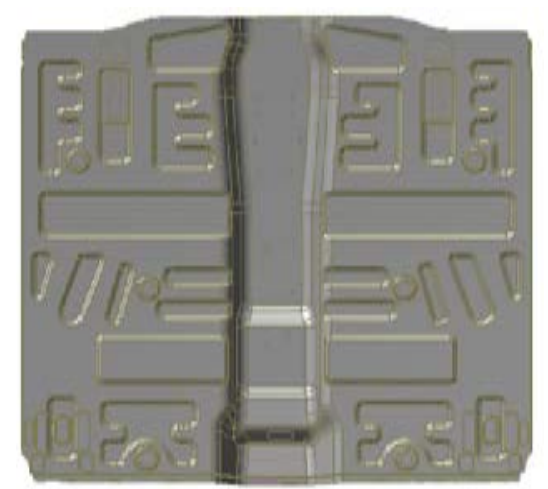

Fig.1: Front floor of car 


\section{VARI process simulation of the front car floor}

Use PAM - RTM to perform simulation calculation on the floor model as shown in figure 1 . The floor is composed of five layers of carbon fiber reinforced body. The basic parameters of materials are shown in table 1 . The resin uses biphenyl $\mathrm{F}$ epoxy resin. The viscosity of resin is an important parameter in the process of simulation and experiment, which will directly affect the molding defects and the

Table1: Basic Parameters in Material

\begin{tabular}{cc}
\hline Basic parameters & Value \\
\hline Resin viscosity & $300 \mathrm{mPa} \bullet \mathrm{s}$ \\
X permeability & $6.2 \times 10^{-11} / \mathrm{m}^{2}$ \\
Y permeability & $6.2 \times 10^{-11} / \mathrm{m}^{2}$ \\
Strengthen body porosity & $62.3 \%$ \\
\hline
\end{tabular}

filling time. In order to be able to get the resin viscosity parameters accurately, this paper adopts rotating viscometer ${ }^{[10]}$ and measured the viscosity as 0.3 Pays. Because the original model of the floor is relatively complex, and there are a lot of characters, in order to perform easy computational simulation, use Catia to get rid of some details, and the floor of the original model is simplified, then put the simplified model of the floor into finite element software Patran for grid partition. Because PAM - RTM software can only perform computing under the trilateral grid, so when the mesh is divided into the trilateral grid to establish finite element model, which is divided into grid type for 2D grid, and the floor division of the grid is shown in figure 2;

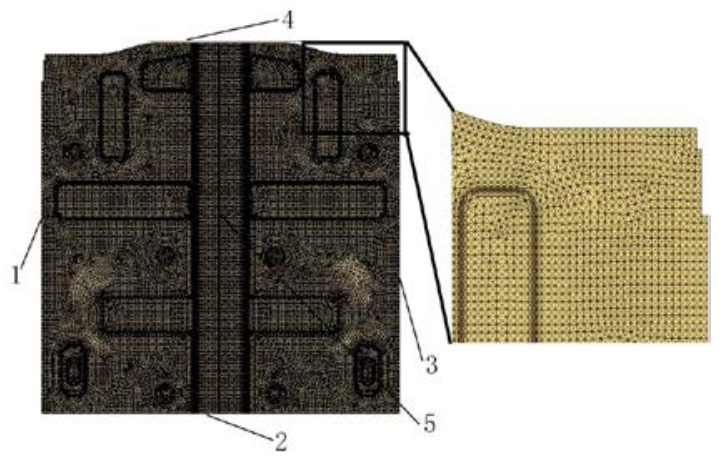

Fig.2: Mesh Generation of the floor

In order to achieve more accurate effect of the simulation, this paper will make more detailed division for the grid, the number of the grid is 104,196, and the number of the node is 52,411 . Finally, imported the finite element model that has meshed into the composite material special simulation software PAMRTM, and set the material parameters and boundary conditions, in which we used three kinds of schemes to do the simulating calculation. The scheme design as shown in Table 2, a main difference three schemes is that the resin injection port differs from the vacuum position. The resin injection location in the third scheme was in the middle axis of symmetry position and vacuum position was on both sides, while the resin injection location and the vacuum position in the first scheme 1 and the second scheme was both located at the side. From figure 3, it can be found that the flow pattern and flow velocity of resin changed with the change of injection mode. The three schemes all are very good to fill the mold without the 


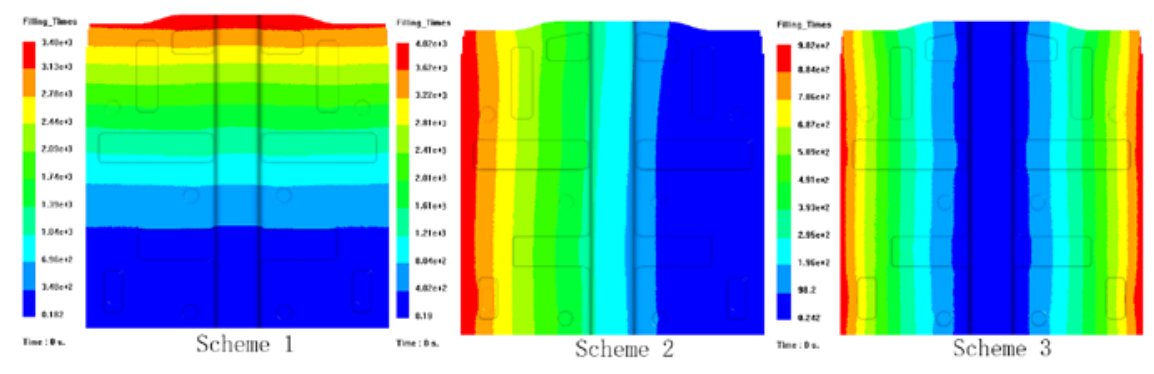

Fig.3: The Filling Time Nephogram for Different Injection Methods

defects like dry spot. However, from the mold filling time of completion of the program can be seen, the filling time of the third scheme is shortest, and the first scheme is longer than the third scheme, the filling time of the second scheme the longest. And the filling time of the third scheme is much shorter than the other two schemes, which is just $28.2 \%$ of the first scheme.

Table2: Forming programs and filling time

\begin{tabular}{|c|c|c|}
\hline Scheme & Resin entrance and vacuum & Filling time (s) \\
\hline \multirow{3}{*}{ I } & Entrance location1 & 3480 \\
\hline & Vacuum position 3 & \\
\hline & Entrance location 2 & 4020 \\
\hline II & Vacuum position 4 & \\
\hline III & $\begin{array}{l}\text { Entrance location } 5 \\
\text { Vacuum position } 1,3\end{array}$ & 982 \\
\hline
\end{tabular}

This could be because of the short distance between the entrance location and export position of resin in the third scheme, over a short distance from the entrance to the resin, so there was less pressure loss during the process for resin from the entrance to the export. While in the first and second scheme, distance between resin entrance location and export position is longer, the resin in the flowing process lost high pressure that leads to the filling time is longer, which can be seen from the pressure profile of the mold filling time in Figure 4.Since the resin injection position and vacuum position with the symmetry axis parallel in scheme 2 and scheme 3 that we can compare the mold filling process in scheme 2 and scheme 3 . The distance for resin to the pumping outlet from the glue injecting mouth in scheme 3 is half of scheme 2, and the filling time is only $24.43 \%$ of scheme 2, it's visible that the increase the flow distance will greatly increase the filling time. The flow in scheme 1 is close to the resin flow in the horizontal plane. Now analyze the scheme 1, Table 3 is the relationship of the range difference between the filling time and the flow front in scheme 1 , and figure 5 is the relation curve of the filling time and the flow front. From which, we can see that the farther distance of resin injection port position, the range

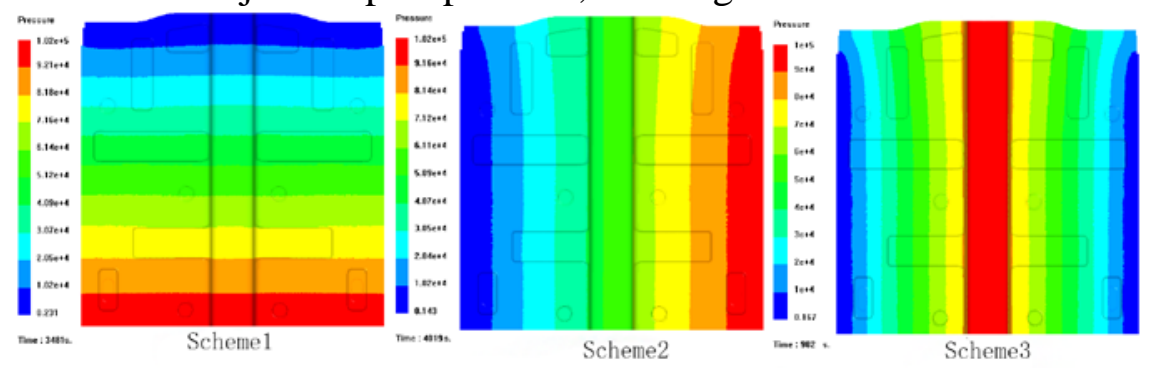

Fig.4:Nephogram of pressure

difference of the flow front is smaller at the same time, and the slope of the curve shown in Figure 5 is smaller as well. This is a description of the reason that the filling time in scheme 1, 2 is far greater than scheme 1. But the specific mathematical relationship between the mold filling time and flow front still needed further study. 
Table3: Relationships between Filling Time and $\Delta \mathrm{x}$ in Program 1

\begin{tabular}{|c|c|c|}
\hline Filling time $/ \mathrm{s}$ & $\begin{array}{l}\text { The Flow } \\
\text { Front/mm }\end{array}$ & $\Delta \mathrm{x} / \mathrm{mm}$ \\
\hline 0 & 0 & 0 \\
\hline 348 & 432 & 432 \\
\hline 696 & 605 & 173 \\
\hline 1044 & 743 & 138 \\
\hline 1392 & 847 & 104 \\
\hline 1741 & 950 & 103 \\
\hline 2089 & 1037 & 87 \\
\hline 2437 & 1114 & 77 \\
\hline 2785 & 1208 & 94 \\
\hline 3133 & 1270 & 62 \\
\hline 3481 & 1321 & 51 \\
\hline \multicolumn{3}{|l|}{1400} \\
\hline \multicolumn{3}{|l|}{1200} \\
\hline \multicolumn{3}{|l|}{1000} \\
\hline \multicolumn{3}{|l|}{800} \\
\hline \multicolumn{3}{|l|}{ 总 600} \\
\hline \multicolumn{3}{|l|}{400} \\
\hline Is 200 & & \\
\hline 0 & $\begin{array}{l}5002000250 \\
\text { ng Time/s }\end{array}$ & 5004000 \\
\hline
\end{tabular}

Fig.5: Relationship between Filling time and the resin flow front

\section{VARI Process Validation Test of Car Front Floor Model}

The filling time of the scheme 3 is the shortest in the three kinds of schemes, and the scheme 3 also is the moulding project what we have to select. To verify the forming of the car floor according to the setting of scheme 3, in which, the selection of materials and simulating calculation is the same to the setting in scheme 3, and the molding test as shown in figure 6 . The test results showed that, the quality of composite floor made in the experiment is good, the scheme does not appear dry spots and other defects, which is consistent with the simulation

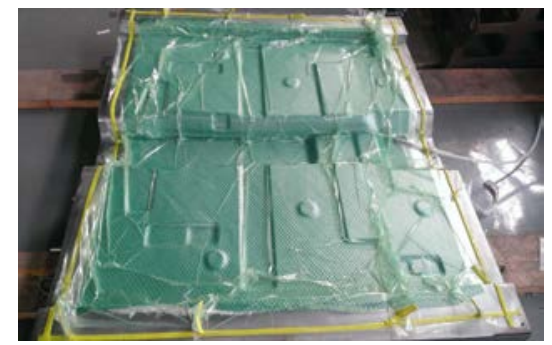

Fig.6: Car Front Floor Molding Test

results. However, the filling time during the process of the experiment is about 820s, and the results of simulation is 982s, which it's shorter compared with the experimental filling time, this could be the result of the following reasons:

(1) In the actual experiment the fiber is paved with the flow medium, so that the flow velocity of resin was accelerated; (2) The permeability around the floor is not the same due to the influence of the edge effect; (3) The structure of the model is larger, and the structure is more complex, due to the uneven artificial press in the laying of reinforcement enhance the porosity and permeability of reinforcement changed; (4) The resin viscosity affected by temperature and the filling time. In this paper, the change of resin viscosity brought by these factors does not take into account, but adopted steady state value.

The process of VARI composites is a very complex process, the process is affected by many factors, these factors in the simulation calculation will be all taken into consideration and also needs 
many research. In this paper, although the mathematical model of the molding process is simplified, some factors not taken into account, the simulation calculation results of mold filling time just more $20 \%$ than the filling time in actual, compared with the actual filling time. Therefore, it can reduce the test times effectively by using the PAM-RTM software to do simulation calculation and save cost, it has important significance for practical production.

\section{Conclusions}

(1) In terms of the thin-walled perform like car front floor that the thickness, length and width are much smaller, can adopt the method of calculating two-dimensional to carry on the simulation, and its the calculation results are close to the actual;

(2) In terms of the forming process of VARI in complex shape parts such as automotive front floor etc. It can get the result that is close to the experiment through the computational simulation, the computational simulation can provide effective reference to experiment;

(3) Compared the three kind of schemes, the filling time of scheme 3 is shortest. Thus in the design of VARI forming process, resin injection port is arranged in the position that is nearest to the location of resin flow terminal. Especially for the symmetrical components, the population flow can be designed near in the axis of symmetry, so that it can mold more easily and fast.

\section{References}

[1] Zhao Qushen , Zhao Panfeng. A Study of VARI [J]. FIBER COMPOSITES, 2002,1: 4546.

[2] Tang Yangge. Simulation Analysis and Verification of 1. 5MW Wind Turbine Blades by Vari [J]. FIBER REINFORCED PLASTICS/COMPOSITES, 2013, 12:30-34

[3] Qiu Jingjing , DUAN Yuexin, LIANG Zhiyong.Computer Simulation and Actual Experiments of RTM Mold-Filling Process Affected by Processing Parameters[J]. Acta Materiae Compositae Sinica, 2004,21 (6) , 70-74. materials, 2004, 21 (6), 70-74.

[4] Zong Hai etc. VARI Simulation of Typical Surface Part and Relevant Experimental Analysis[J].POLYMER MAT ERIALS SCIENCE AND ENGINEERING, 2010, 26 (11): 156159., 2010,26 (11) : 156-159.

[5] Lu Chang.Comparison Between Numerical and Experimental Results for Mold Filing in Resin Transfer Molding[J].Acta Materiae Compositae Sinica 1999,16(1):132-136.

[6] Zhang Shaochun Lu Guanxing Huang Yizhang etc.RTM Filling Process Numerical Simulation[J]. FIBER REINFORCED PLASTICS/ COMPOSITES , 1996,6:1-5.

[7] DENG Jing-lan,WANG Ji-hui,LIAN Jun . Computer Simulation of RTM Mold Filling Process( I ): Mathematic Model[J] . JOURNAL OF WUHAN UNIVERSITY OF TECHNOLOGY, 2003, 25(7) :51-53.

[8] Gerd M,Sven B,Hugo S.2D permeability tensor identification of fibrous reinforcements for RTM using an inverse method [J]. Composite Part A,2008, 39(9):1530-1536.

[9] Jiang Shunliang. SIMULATION FOR RTM MOLD FILLING[J].Acta Materiae Compositae Sinica ,2002,19 (2):13-17.

[10] Cheng Tianjian etc..Research on Composite Reinforced Fiber Permeability Measuring andVari Process Simulate[J].Engineering Plastics, 2011,39 (7) : 38-40. 